\title{
Classical gluon production amplitude in heavy-ion collisions
}

\author{
Giovanni Antonio Chirilli ${ }^{1,2, \star}$ \\ ${ }^{1}$ Institut für Theoretische Physik, University of Regensburg, D-93040 Regensburg, Germany
}

\begin{abstract}
The initial condition for the formation of the Quark-Gluon Plasma, the distribution of quarks and gluons produced in the initial stages of nuclear collisions, is the fundamental building block of heavy-ion theory. In the framework of saturation physics and Wilson lines formalism we calculate the scattering amplitude, beyond the leading order, of the classical gluon produced in heavy-ion collisions.

known as the initial condition of the Quark-Gluon Plasma formation, is the fundamental building block of heavy-ion theory.
\end{abstract}

\section{Introduction}

To understand the initial stages of nuclear collisions and the formation of quark gluon plasma, one has to study the dynamics of the collisions from first principle in Quantum Chromodynamic (QCD). To this end, we calculate the scattering amplitude, beyond the leading order, of the classical gluon produced in Nucleus-Nucleus collisions.

As a first approximation, we study the collision of two large nuclei with atomic number $1 \ll A_{1} \ll$ $A_{2}$. The smaller nucleus can be treated as a diluted system of constituents when compared to the large nucleus. In particular, the saturation scale of nucleus $A_{1}$, which is proportional to its atomic number, $Q_{s 1}^{2} \sim \alpha_{s}^{2} A_{s 1}^{1 / 3}$ is much smaller then the saturation scale of the large nucleus, $Q_{2 s}^{2} \sim \alpha_{s}^{2} A_{2}^{1 / 3}$. The atomic number of the nucleus enters in the resummation parameter. Indeed, if we consider a particle propagation through a large nucleus with atomic number $A$, with good probability, will encounter, along its path, $A^{1 / 3}$ nucleons. In the two-gluon approximation, the particle propagating through the nucleus, interacts with each nucleon with the exchange of two gluons. Hence, for large nuclei, there are contributions to the scattering amplitude that are proportional to $\alpha_{s}^{2} A^{1 / 3}$ and since $A$ is a large number, these contributions are the leading ones. In our case we have $\alpha_{s}^{2} A_{2}^{1 / 3} \sim 1$ for the larger nucleus (the target) which in the boosted Lorentz frame reduces to a shock-wave (represented as a red stripe in all diagrams below), and $\alpha_{s}^{2} A_{1}^{1 / 3} \leq 1$ for the smaller one (the projectile).

\section{From proton-Nucleus to Heavy-light Ion Collisions}

The leading-order (LO) contribution to the classical gluon production amplitude in heavy-light ions collisions coincides with proton-Nucleus ( $\mathrm{pA}$ ) collisions. The diagrams for $\mathrm{pA}$ collisions are shown in Fig. 1. Using light-cone coordinates $x^{ \pm}=\frac{1}{\sqrt{2}}\left(x^{0} \pm x^{3}\right)$, the proton propagates along $x^{+}$direction and

^e-mail: giovanni.chirilli@physik.uni-regensburg.de 

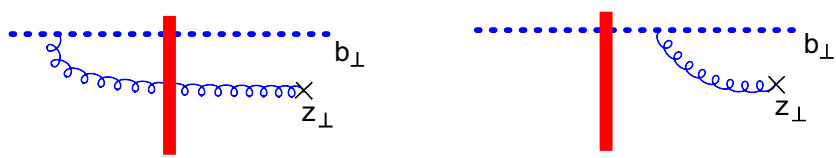

Figure 1. Feynman diagrams for pA collisions. Dashed lines represent Wilson lines.

the target (large nucleus) in $x^{-}$direction. Transverse coordinate are $\vec{x}_{\perp}=\left(x^{1}, x^{2}\right)$. We will perform the calculation in the $A^{+}=0$ light-cone gauge. Note that the blue dashed lines represent Wilson lines. The scattering amplitude for $\mathrm{pA}$ collision is [1]

$$
A\left(\vec{z}_{\perp}, \vec{b}_{\perp}\right)=\frac{i g}{\pi} \frac{\vec{\epsilon}_{\perp}^{\lambda *} \cdot\left(\vec{z}_{\perp}-\vec{b}_{\perp}\right)}{\left|\vec{z}_{\perp}-\vec{b}_{\perp}\right|^{2}}\left[U_{\vec{z}_{\perp}}^{a b}-U_{\vec{b}_{\perp}}^{a b}\right]\left(V_{\vec{b}_{\perp}} t^{b}\right)
$$

where $\vec{\epsilon}_{\perp}^{*}$ is the polarization vector of the emitted gluon, $U_{\vec{z}_{\perp}}^{a b}$ is the Wilson line in the adjoint representation at transverse position $\vec{z}_{\perp}$, while $V_{\vec{b}}$ is the Wilson line in the fundamental representation. The gluon production cross section is proportional to the amplitude square and is given by

$$
\frac{d \sigma}{d^{2} k_{T} d y}=\frac{1}{2(2 \pi)^{3}} \int d^{2} z d^{2} z^{\prime} d^{2} b e^{-i \vec{k}_{\perp} \cdot\left(\vec{z}_{\perp}-\vec{z}_{\perp}^{\prime}\right)}\left\langle A\left(\vec{z}_{\perp}, \vec{b}_{\perp}\right) A^{*}\left(\vec{z}_{\perp}^{\prime}, \vec{b}_{\perp}\right)\right\rangle
$$

We are interested in calculating the next-to-leading order (NLO) contribution to the gluon production cross-section which is proportional to $\alpha_{s}^{3} A_{1}^{2 / 3}$. The diagrams that contribute at this order may be divided in three classes as shown in Fig. 2 [2]. It is possible to reduce dramatically the number of

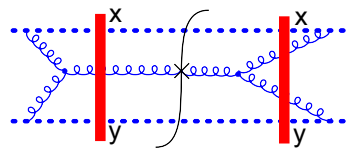

(a)

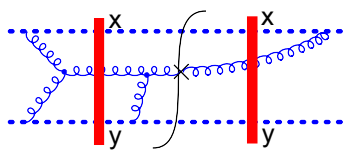

(b)

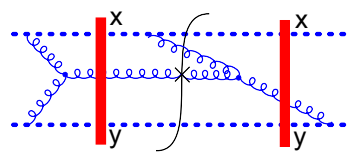

(c)

Figure 2. Here we show the three different classes of diagrams at order $g^{6}$ : (a) Square of order $g^{3}$ amplitudes; (b) Interference between order $g^{5}$ and order $g$ amplitudes; (c) Interference between order $g^{4}$ and order $g^{2}$ amplitudes.

diagrams if we sum up diagrams having gluons on one side of the cut with the corresponding diagrams having gluons crossing the cut, thus reducing the two diagrams in a single diagram with a retarded gluon propagator (see Fig.3)

$$
\frac{-i d^{\mu \nu}(k)}{k^{2}+i \epsilon}+2 \pi \theta\left(-k^{+}\right) \delta\left(k^{2}\right) d^{\mu \nu}(k)=\frac{-i d^{\mu \nu}(k)}{k^{2}+i \epsilon k^{+}}
$$




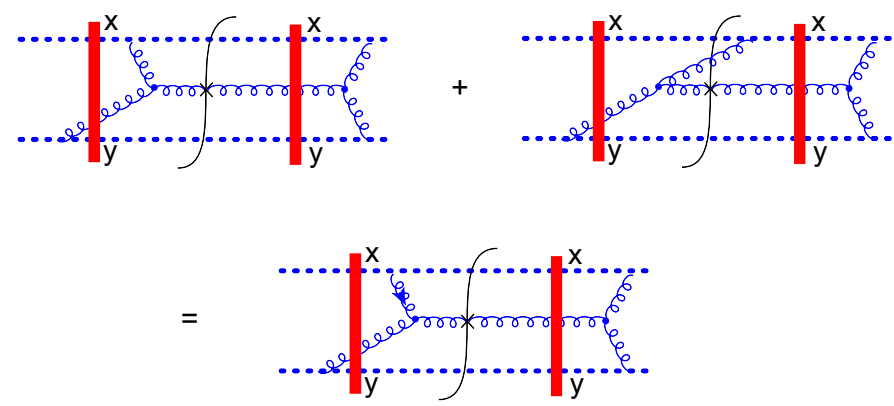

Figure 3. Summing up diagrams with gluons on one side of the cut with the corresponding diagram with gluons crossing the cut, we get a single diagram with a retarded gluon propagator.

where $d^{\mu v}(k)=g^{\mu \nu}-\frac{\eta^{\mu} k^{v}+\eta^{\nu} k^{\mu}}{\eta \cdot k}$. A consequence of this observation is that, the only diagrams we will have to consider are those at order $g^{3}$ and those at order $g^{5}$ (at amplitude level), but with each gluon propagator being retarded propagator.

A further reduction of the number of diagrams to be calculated is given by the following two observations. First, we notice that the sum of diagrams of the type in Fig. 5 cancel. Second, using the

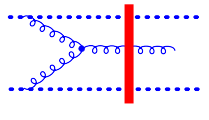

$\mathrm{A}_{1}$
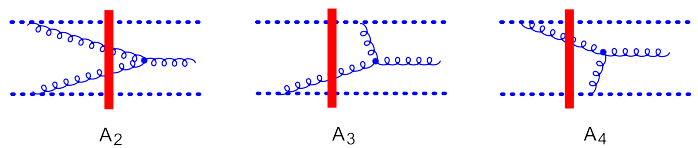

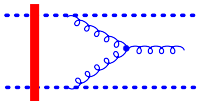

$A_{5}$

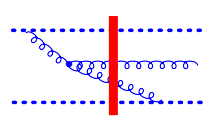

$A_{6}$

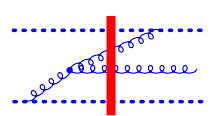

$\mathrm{A}_{7}$

Figure 4. Diagrams A have single 3-gluon vertex with gluons connecting to both quarks. All gluons are retarded and the time flows to the right.

cancellation described in Fig. 5 we can combine diagrams as shown in Fig. 6 thus reducing further the number of diagrams (see Fig. 7).

The result for the calculation of diagrams in Fig. 4 and in Fig. 7 is [2]

$$
\begin{aligned}
& \sum_{i=1}^{7} A_{i}+\sum_{i=1}^{12} B_{i}+\sum_{i=1}^{12} C_{i}=-\frac{g^{3}}{4 \pi^{4}} \int d^{2} x_{1} d^{2} x_{2} \delta\left[\left(\vec{z}_{\perp}-\vec{x}_{1 \perp}\right) \times\left(\vec{z}_{\perp}-\vec{x}_{2 \perp}\right)\right] \\
& \times\left[\frac{\vec{\epsilon}_{\perp}^{\lambda *} \cdot\left(\vec{x}_{2 \perp}-\vec{x}_{1 \perp}\right)}{\left|\vec{x}_{2 \perp}-\vec{x}_{1 \perp}\right|^{2}} \frac{\vec{x}_{1 \perp}-\vec{b}_{1 \perp}}{\left|\vec{x}_{1 \perp}-\vec{b}_{1 \perp}\right|^{2}} \cdot \frac{\vec{x}_{2 \perp}-\vec{b}_{2 \perp}}{\left|\vec{x}_{2 \perp}-\vec{b}_{2 \perp}\right|^{2}}-\frac{\vec{\epsilon}_{\perp}^{\lambda *} \cdot\left(\vec{x}_{1 \perp}-\vec{b}_{1 \perp}\right)}{\left|\vec{x}_{1 \perp}-\vec{b}_{1 \perp}\right|^{2}} \frac{\vec{z}_{\perp}-\vec{x}_{1 \perp}}{\left|\vec{z}_{\perp}-\vec{x}_{1 \perp}\right|^{2}} \cdot \frac{\vec{x}_{2 \perp}-\vec{b}_{2 \perp}}{\left|\vec{x}_{2 \perp}-\vec{b}_{2 \perp}\right|^{2}}\right. \\
& \left.+\frac{\vec{\epsilon}_{\perp}^{\lambda *} \cdot\left(\vec{x}_{2 \perp}-\vec{b}_{2 \perp}\right)}{\left|\vec{x}_{2 \perp}-\vec{b}_{2 \perp}\right|^{2}} \frac{\vec{x}_{1 \perp}-\vec{b}_{1 \perp}}{\left|\vec{x}_{1 \perp}-\vec{b}_{1 \perp}\right|^{2}} \cdot \frac{\vec{z}_{\perp}-\vec{x}_{2 \perp}}{\left|\vec{z}_{\perp}-\vec{x}_{2 \perp}\right|^{2}}\right] \times
\end{aligned}
$$




$$
\begin{aligned}
& \times f^{a b c}\left[U_{\vec{x}_{1 \perp}}^{b d}-U_{\vec{b}_{1 \perp}}^{b d}\right]\left[U_{\vec{x}_{2 \perp}}^{c e}-U_{\vec{b}_{2 \perp}}^{c e}\right]\left(V_{\vec{b}_{1 \perp}} t^{d}\right)_{1}\left(V_{\vec{b}_{2 \perp}} t^{e}\right)_{2}+\frac{i g^{3}}{4 \pi^{3}} f^{a b c}\left(V_{\vec{b}_{1 \perp}} t^{d}\right)_{1}\left(V_{\vec{b}_{2 \perp}} t^{e}\right)_{2} \\
& \times \int d^{2} x\left[U _ { \vec { b } _ { 1 \perp } } ^ { b d } ( U _ { \vec { x } _ { \perp } } ^ { c e } - U _ { \vec { b } _ { 2 \perp } } ^ { c e } ) \left(\frac{\vec{\epsilon}_{\perp}^{\lambda *} \cdot\left(\vec{z}_{\perp}-\vec{x}_{\perp}\right)}{\left|\vec{z}_{\perp}-\vec{x}_{\perp}\right|^{2}} \frac{\vec{x}_{\perp}-\vec{b}_{1 \perp}}{\left|\vec{x}_{\perp}-\vec{b}_{1 \perp}\right|^{2}} \cdot \frac{\vec{x}_{\perp}-\vec{b}_{2 \perp}}{\left|\vec{x}_{\perp}-\vec{b}_{2 \perp}\right|^{2}}\right.\right. \\
& \left.-\frac{\vec{\epsilon}_{\perp}^{\lambda *} \cdot\left(\vec{z}_{\perp}-\vec{b}_{1 \perp}\right)}{\left|\vec{z}_{\perp}-\vec{b}_{1 \perp}\right|^{2}} \frac{\vec{z}_{\perp}-\vec{x}_{\perp}}{\left|\vec{z}_{\perp}-\vec{x}_{\perp}\right|^{2}} \cdot \frac{\vec{x}_{\perp}-\vec{b}_{2 \perp}}{\left|\vec{x}_{\perp}-\vec{b}_{2 \perp}\right|^{2}}-\frac{\vec{\epsilon}_{\perp}^{\lambda *} \cdot\left(\vec{z}_{\perp}-\vec{b}_{1 \perp}\right)}{\left|\vec{z}_{\perp}-\vec{b}_{1 \perp}\right|^{2}} \frac{\vec{x}_{\perp}-\vec{b}_{1 \perp}}{\left|\vec{x}_{\perp}-\vec{b}_{1 \perp}\right|^{2}} \cdot \frac{\vec{x}_{\perp}-\vec{b}_{2 \perp}}{\left|\vec{x}_{\perp}-\vec{b}_{2 \perp}\right|^{2}}\right) \\
& -\left(U_{\vec{x}_{\perp}}^{b d}-U_{\vec{b}_{1 \perp}}^{b d}\right) U_{\vec{b}_{2 \perp}}^{c e}\left(\frac{\vec{\epsilon}_{\perp}^{\lambda *} \cdot\left(\vec{z}_{\perp}-\vec{x}_{\perp}\right)}{\left|\vec{z}_{\perp}-\vec{x}_{\perp}\right|^{2}} \frac{\vec{x}_{\perp}-\vec{b}_{1 \perp}}{\left|\vec{x}_{\perp}-\vec{b}_{1 \perp}\right|^{2}} \cdot \frac{\vec{x}_{\perp}-\vec{b}_{2 \perp}}{\left|\vec{x}_{\perp}-\vec{b}_{2 \perp}\right|^{2}}\right. \\
& \left.\left.-\frac{\vec{\epsilon}_{\perp}^{\lambda *} \cdot\left(\vec{z}_{\perp}-\vec{b}_{2 \perp}\right)}{\left|\vec{z}_{\perp}-\vec{b}_{2 \perp}\right|^{2}} \frac{\vec{z}_{\perp}-\vec{x}_{\perp}}{\left|\vec{z}_{\perp}-\vec{x}_{\perp}\right|^{2}} \cdot \frac{\vec{x}_{\perp}-\vec{b}_{1 \perp}}{\left|\vec{x}_{\perp}-\vec{b}_{1 \perp}\right|^{2}}-\frac{\vec{\epsilon}_{\perp}^{\lambda *} \cdot\left(\vec{z}_{\perp}-\vec{b}_{2 \perp}\right)}{\left|\vec{z}_{\perp}-\vec{b}_{2 \perp}\right|^{2}} \frac{\vec{x}_{\perp}-\vec{b}_{1 \perp}}{\left|\vec{x}_{\perp}-\vec{b}_{1 \perp}\right|^{2}} \cdot \frac{\vec{x}_{\perp}-\vec{b}_{2 \perp}}{\left|\vec{x}_{\perp}-\vec{b}_{2 \perp}\right|^{2}}\right)\right] \\
& -\frac{i g^{3}}{4 \pi^{2}} f^{a b c}\left(V_{\vec{b}_{1 \perp}} t^{d}\right)_{1}\left(V_{\vec{b}_{2 \perp}} t^{e}\right)_{2}\left[\left(U_{\vec{z}_{\perp}}^{b d}-U_{\vec{b}_{1 \perp}}^{b d}\right) U_{\vec{b}_{2 \perp}}^{c e} \frac{\vec{\epsilon}_{\perp}^{\lambda *} \cdot\left(\vec{z}_{\perp}-\vec{b}_{1 \perp}\right)}{\left|\vec{z}_{\perp}-\vec{b}_{1 \perp}\right|^{2}} \ln \frac{1}{\left|\vec{z}_{\perp}-\vec{b}_{2 \perp}\right| \Lambda}-U_{\vec{b}_{1 \perp}}^{b d}\left(U_{\vec{z}_{\perp}}^{c e}\right.\right. \\
& \left.\left.-U_{\vec{b}_{2 \perp}}^{c e}\right) \frac{\vec{\epsilon}_{\perp}^{\lambda *} \cdot\left(\vec{z}_{\perp}-\vec{b}_{2 \perp}\right)}{\left|\vec{z}_{\perp}-\vec{b}_{2 \perp}\right|^{2}} \ln \frac{1}{\left|\vec{z}_{\perp}-\vec{b}_{1 \perp}\right| \Lambda}\right]-\frac{i g^{3}}{4 \pi^{3}} \int d^{2} x\left[U_{x_{\perp}}^{a b}-U_{z_{\perp}}^{a b}\right] f^{b d e}\left(V_{\vec{b}_{1 \perp}} t^{d}\right)_{1}\left(V_{\vec{b}_{2 \perp}} t^{e}\right)_{2} \\
& \times \frac{\vec{\epsilon}_{\perp}^{\lambda *} \cdot\left(\vec{z}_{\perp}-\vec{x}_{\perp}\right)}{\left|\vec{z}_{\perp}-\vec{x}_{\perp}\right|^{2}} \frac{\vec{x}_{\perp}-\vec{b}_{1 \perp}}{\left|\vec{x}_{\perp}-\vec{b}_{1 \perp}\right|^{2}} \cdot \frac{\vec{x}_{\perp}-\vec{b}_{2 \perp}}{\left|\vec{x}_{\perp}-\vec{b}_{2 \perp}\right|^{2}} \operatorname{Sign}\left(b_{2}^{-}-b_{1}^{-}\right) .
\end{aligned}
$$

Another class of diagrams that contribute at $g^{5}$ order is shown in Fig. 8 and the result is [2]

$$
\begin{aligned}
& \sum_{i=1}^{6} D_{i} \\
= & -\frac{g^{3}}{8 \pi^{4}} \int d^{2} x_{1} d^{2} x_{2} \delta\left[\left(\vec{z}_{\perp}-\vec{x}_{1 \perp}\right) \times\left(\vec{z}_{\perp}-\vec{x}_{2 \perp}\right)\right]\left[\frac{\vec{\epsilon}_{\perp}^{\lambda *} \cdot\left(\vec{x}_{2 \perp}-\vec{x}_{1 \perp}\right)}{\left|\vec{x}_{2 \perp}-\vec{x}_{1 \perp}\right|^{2}} \frac{\vec{x}_{1 \perp}-\vec{b}_{2 \perp}}{\left|\vec{x}_{1 \perp}-\vec{b}_{2 \perp}\right|^{2}} \cdot \frac{\vec{x}_{2 \perp}-\vec{b}_{2 \perp}}{\left|\vec{x}_{2 \perp}-\vec{b}_{2 \perp}\right|^{2}}\right. \\
& \left.-\frac{\vec{\epsilon}_{\perp}^{\lambda *} \cdot\left(\vec{x}_{1 \perp}-\vec{b}_{2 \perp}\right)}{\left|\vec{x}_{1 \perp}-\vec{b}_{2 \perp}\right|^{2}} \frac{\vec{z}_{\perp}-\vec{x}_{1 \perp}}{\left|\vec{z}_{\perp}-\vec{x}_{1 \perp}\right|^{2}} \cdot \frac{\vec{x}_{2 \perp}-\vec{b}_{2 \perp}}{\left|\vec{x}_{2 \perp}-\vec{b}_{2 \perp}\right|^{2}}+\frac{\vec{\epsilon}_{\perp}^{\lambda *} \cdot\left(\vec{x}_{2 \perp}-\vec{b}_{2 \perp}\right)}{\left|\vec{x}_{2 \perp}-\vec{b}_{2 \perp}\right|^{2}} \frac{\vec{x}_{1 \perp}-\vec{b}_{2 \perp}}{\left|\vec{x}_{1 \perp}-\vec{b}_{2 \perp}\right|^{2}} \cdot \frac{\vec{z}_{\perp}-\vec{x}_{2 \perp}}{\left|\vec{z}_{\perp}-\vec{x}_{2 \perp}\right|^{2}}\right] \\
& \times f^{a b c}\left[U_{\vec{x}_{1 \perp}}^{b d}-U_{\vec{b}_{2 \perp}}^{b d}\right]\left[U_{\vec{x}_{2 \perp}}^{c e}-U_{\vec{b}_{2 \perp}}^{c e}\right]\left(V_{\vec{b}_{1 \perp}}\right)_{1}\left(V_{\vec{b}_{2 \perp}} t^{e} t^{d}\right)_{2} \\
& +\frac{i g^{3}}{4 \pi^{3}} \int d^{2} x f^{a b c} U_{\vec{b}_{2 \perp}}^{b d}\left[U_{\vec{x}_{\perp}}^{c e}-U_{\vec{b}_{2 \perp}}^{c e}\right]\left(V_{\vec{b}_{1 \perp}}\right)_{1}\left(V_{\vec{b}_{2 \perp}} t^{e} t^{d}\right)_{2}\left(\frac{\vec{\epsilon}_{\perp}^{\lambda *} \cdot\left(\vec{z}_{\perp}-\vec{x}_{\perp}\right)}{\left|\vec{z}_{\perp}-\vec{x}_{\perp}\right|^{2}} \frac{1}{\left|\vec{x}_{\perp}-\vec{b}_{2 \perp}\right|^{2}}\right. \\
& \left.-\frac{\vec{\epsilon}_{\perp}^{\lambda *} \cdot\left(\vec{z}_{\perp}-\vec{b}_{2 \perp}\right)}{\vec{z}_{\perp}-\vec{x}_{\perp}} \cdot \frac{\vec{x}_{\perp}-\vec{b}_{2 \perp}}{\left|\vec{z}_{2 \perp}\right|^{2}-\left.\vec{x}_{\perp}\right|^{2}} \frac{\vec{\epsilon}_{\perp}^{\lambda *} \cdot\left(\vec{z}_{\perp}-\vec{b}_{2 \perp}\right)}{\left|\vec{x}_{\perp}-\vec{b}_{2 \perp}\right|^{2}} \frac{1}{\left|\vec{z}_{2 \perp}\right|^{2}-\left.\vec{b}_{2 \perp}\right|^{2}}\right) \\
& +\frac{i g^{3}}{4 \pi^{2}} f^{a b c} U_{\vec{b}_{2 \perp}}^{b d}\left[U_{\vec{z}_{\perp}}^{c e}-U_{\vec{b}_{2 \perp}}^{c e}\right]\left(V_{\vec{b}_{1 \perp}}\right)_{1}\left(V_{\vec{b}_{2 \perp}} t^{e} t^{d}\right)_{2} \frac{\vec{\epsilon}_{\perp}^{\lambda *} \cdot\left(\vec{z}_{\perp}-\vec{b}_{2 \perp}\right)}{\left|\vec{z}_{\perp}-\vec{b}_{2 \perp}\right|^{2}} \ln \frac{1}{\left|\vec{z}_{\perp}-\vec{b}_{2 \perp}\right| \Lambda} .
\end{aligned}
$$

\section{Gauge invariance}

As we stated above, the calculation was carried out in the light-cone gauge. It is well known that the light-cone gauge posses an ambiguity about the light-cone singularity $k^{+}=0$. In Ref. [3] we showed that this ambiguity is related to the residual sub-gauge choice, and that different prescriptions 


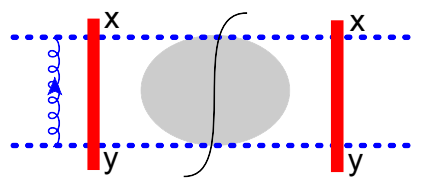

(a)

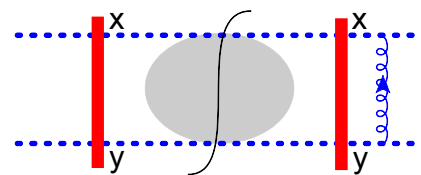

(b)

Figure 5. The shaded region represents a general interaction. The two diagrams, with retarded gluon propagators with time flow in the direction of the arrow, cancel.
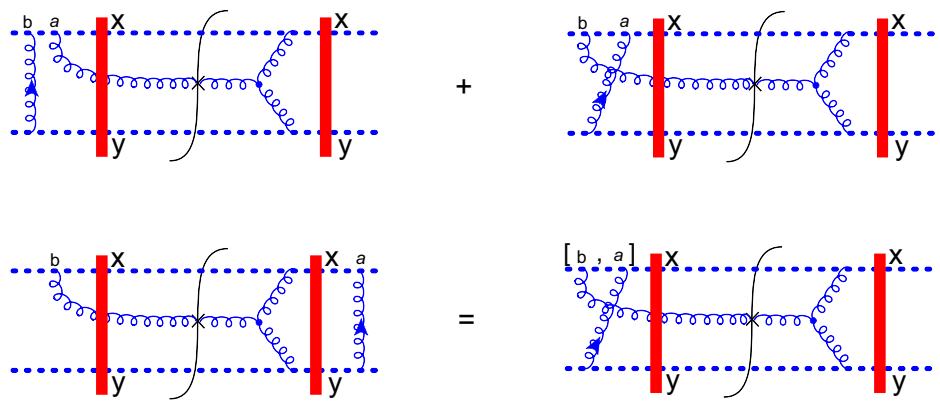

Figure 6. The sum of these three diagrams is equal to the diagram with the color factor associated with the top quark replaced by a commutator. The commutator is represented by a bracket, [, ].
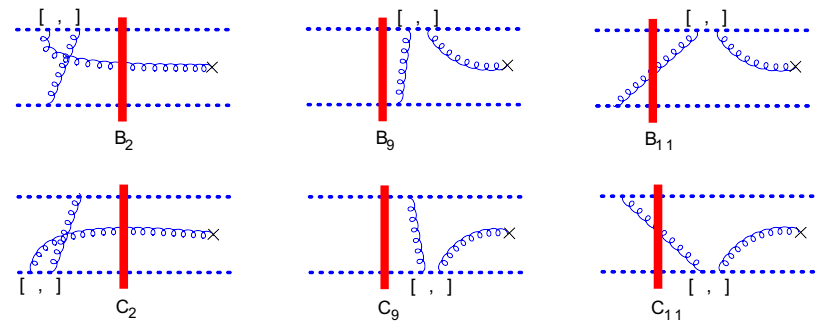

Figure 7. Diagrams B and Diagrams C consist of a single gluon emission with one gluon traveling between the quarks. The color factor associated with the top quark has been replaced with a commutator. The gluons connecting the quarks are retarded with the momentum flowing to the top quark line for diagrams B, and retarded with momentum flowing to the bottom quark line for diagrams $\mathrm{C}$.

for the $k^{+}$singularity coincide with different sub-gauge conditions. It turned out that the quark lines reduce to Wilson lines if we regulate the light-cone singularity with Principle-Value (PV). The subgauge condition that leads to PV-prescription is $\partial_{\perp} \cdot A_{\perp}\left(x^{-}=+\infty\right)+\partial_{\perp} \cdot A_{\perp}\left(x^{-}=-\infty\right)=0$ and the light-cone propagator is

$$
D_{P V}^{\mu v}(x, y) \equiv \int \frac{d^{4} k}{(2 \pi)^{4}} e^{-i k \cdot(x-y)} \frac{-i}{k^{2}+i \epsilon}\left[g_{\perp}^{\mu \nu}-\left(k_{\perp}^{\mu} \eta^{v}+k_{\perp}^{v} \eta^{\mu}\right) \mathrm{PV}\left\{\frac{1}{k^{+}}\right\}-2 \eta^{\mu} \eta^{v} k^{-} \mathrm{PV}\left\{\frac{1}{k^{+}}\right\}\right]
$$



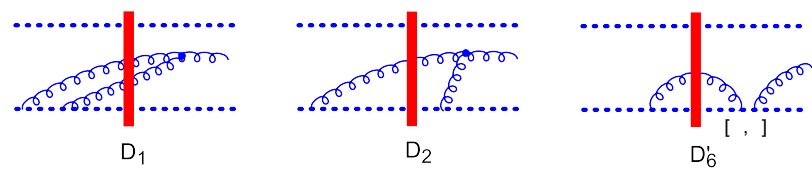

Figure 8. Diagrams involving only one quark at the amplitude level. All gluons are retarded with momentum flowing to the right.

where $\mathrm{PV}\left\{\frac{1}{k^{+}}\right\} \equiv \frac{1}{2}\left(\frac{1}{k^{+}+i \epsilon}+\frac{1}{k^{+}-i \epsilon}\right)$. Alternatively, one may choose sub-gauge condition $\vec{\partial}_{\perp} \cdot \vec{A}_{\perp}\left(x^{-}=\right.$ $+\infty)=0$ and the propagator is

$$
D_{1}^{\mu v}(x, y) \equiv \int \frac{d^{4} k}{(2 \pi)^{4}} e^{-i k \cdot(x-y)} \frac{-i}{k^{2}+i \epsilon}\left[g^{\mu \nu}-\frac{k^{\mu} \eta^{v}}{k^{+}-i \epsilon}-\frac{k^{v} \eta^{\mu}}{k^{+}+i \epsilon}\right]
$$

For sub-gauge condition at $x^{-}=-\infty$ i.e $\vec{\partial}_{\perp} \cdot \vec{A}_{\perp}\left(x^{-}=-\infty\right)=0$ the propagator is

$$
D_{2}^{\mu \nu}(x, y) \equiv \int \frac{d^{4} k}{(2 \pi)^{4}} e^{-i k \cdot(x-y)} \frac{-i}{k^{2}+i \epsilon}\left[g^{\mu v}-\frac{k^{\mu} \eta^{v}}{k^{+}+i \epsilon}-\frac{k^{v} \eta^{\mu}}{k^{+}-i \epsilon}\right] .
$$

The results we obtained in Ref. [2] is shown to be invariant under the above sub-gauge choices.

\section{Conclusion and Outlook}

The results presented here represent a first step toward obtaining the single inclusive gluon production cross-section for heavy-light ions collisions at the classical level beyond the LO. The results we obtained are reasonably compact considering the number of diagrams that contribute at this order. For the success of this calculation it was important to organize the diagrams in different classes and to combine many of them to fewer effective diagrams.

It is certainly desirable to compare our result to the similar one obtained in Ref. [4], but such comparison will not be possible until all the diagrams of order $g^{5}$ (at amplitude level) will be calculated. Moreover, since our result is at the classical level and there are no quantum fluctuations, it also desirable to check its conformal invariance in the transverse coordinate space. However, at this point this check cannot be performed since diagrams at order $g^{5}$ at the amplitude level have still to be calculated.

The author is grateful to the organizers of QCD@work 2016 for warm hospitality.

\section{References}

[1] Y. V. Kovchegov and A. H. Mueller, Gluon production in current nucleus and nucleon nucleus collisions in a quasi-classical approximation, Nucl. Phys. B529 (1998) 451-479 [hep-ph/9802440].

[2] G. A. Chirilli, Y. V. Kovchegov, D. E. Wertepny, Classical Gluon Production Amplitude for Nucleus-Nucleus Collisions: First Saturation Correction in the Projectile, JHEP 1513 (2015) 015 [arxiv: 1501.03106].

[3] G. A. Chirilli, Y. V. Kovchegov and D. E. Wertepny, JHEP 1512, 138 (2015) doi:10.1007/JHEP12(2015)138 [arXiv:1508.07962 [hep-ph]].

[4] I. Balitsky, Scattering of shock waves in QCD, Phys. Rev. D70 (2004) 114030 [hep-ph/0409314]. 